\title{
Threshold exposure duration as a function of luminance for visual recognition
}

\author{
KATHERINE E. BAKER \\ UNIVERSITY OF NEBRASKA 1
}

\begin{abstract}
Threshold exposure duration was measured at set luminances for recognition at successively higher criteria of $1,2,3,4$, and 5 correct letters in nonsense words. Two sizes of letters were used and triads of Landolt " $C s$ " were compared with letters. Tachistoscopic procedures were the typical ascending series in the method of limits and pre- and postexposure light adaptation. Obtained $\log t$ vs log I functions were two-part straight line curves both describable by the equation, $\log t+\log I^{k}=\log C$, but with $k$ and $C$ abruptly changing at a certain I. Reciprocity $(k=1)$ held only for low Is and the low criterion; as criterion was raised at low Is $k$ became less negative (about .60 at five correct) for both sizes and for "Cs." At higher Is, $k$ shifted from .25 to -.40 with increasing criterion for large letters, but varied around .45 at all criteria for small. The lower the criterion, the lower the I and $t$ at which $k$ and $C$ abruptly changed for large letters, but only $t$ was lower for small. Changes in $t$ with acuity requirement change, and from one criterion level to the next, were not uniform over all Is. Results may reflect obscuring procedural factors or basic properties of visual recognition processes. Some interpretations in terms of procedural variables are offered. It appears more likely that visual recognition involves central processes for which time is needed in excess of that for summation of luminances for mere detection by sensory processes, as expressed in Bloch's reciprocity law.
\end{abstract}

The prevalent use of duration thresholds for visual recognition of words, and other stimulus patterns, demands both more empirical information and more discussion of interpretation of intensity-time relations for such perceptual tasks. Recognition generally involves durations well above $.01 \mathrm{sec}$, but less than $1 \mathrm{sec}$, under the luminance level and other conditions of the experiments. However, exposure duration/luminance relationships in visual perception tasks require cautious interpretation in relation to a variety of events contributing to the process under investigation.

Visual recognition depends upon initial sensory events for brightness discrimination and resolution of details. For these initial responses there is reciprocity of intensity and time below a critical duration, $t_{c}$, of about .01-.02 sec (Bunsen-Roscoe or Bloch law). All types of acuity have exhibited reciprocity up to a $t_{c}$, beyond which duration no longer has an effect (Graham \& Cook, 1937; Riggs, 1965; Lit, 1968). Graham and Ratoosh (1962) argue convincingly that durations in excess of $t_{c}$ do not apply to initial sensory events, that longer durations cannot alter already past initial events, but must imply time determinants of other processes entering into the total process. Some question has arisen, however, concerning the appropriate value for $t_{c}$ to represent the limits of perfect summation for purely sensory events. Visual acuity measures, as well as span of apprehension data (Hunter \& Sigler, 1940), show reciprocity up to $.30 \mathrm{sec}$ or more and would appear to indicate quite high $t_{c} s$ in certain cases. But Kahneman and Norman (1964) and also Neisser (1967) suggest that processes subsequent to initial sensory ones, presumed to have a $t_{c}$ no higher than $.05-.10 \mathrm{sec}$, may also exhibit reciprocity relations over much longer durations and, thus, these higher limiting durations have different implications.

Neisser's (1967) major emphasis is that stimulus duration underestimates the duration of another early perceptual process of prime importance. Sensory events give rise to what he terms iconic memory, a short-term perceptual memory process that considerably outlasts stimulus exposure and serves as the more immediate precursor of perceptual recognition behavior. Intensity-time relations for stimuli are of concern since these variables influence duration of iconic memory. Neisser's conclusions from relevant experiments are: (1) Iconic memory endures about $5 \mathrm{sec}$ if the postexposure field is dark, but only $1 \mathrm{sec}$ if bright. (2) Stimulus intensity and time determine duration of the subsequent icon until its critical duration is reached. (3) Perfect summation may well hold up to quite long durations where duration of icon is concerned. Neisser's view includes a more permanent coding of information contained in the icon to permit verbal reporting; this coding must be done before the icon fades and is influenced by several additional variables.

Tachistoscopic studies of word recognition do not of ten reflect appreciation of these complex questions. Different studies employ different luminance levels that are frequently unspecified; often data are $\mathrm{z}$ scores, not time; generally luminance is held constant, making assessment of its influence impossible. Unfortunately, data from such studic ; cannot be interpreted in the light of the refined intensity-time investigations since the latter, for well-considered reasons, use markedly different procedures. As a preliminary step, clear empirical information is needed on functions relating duration thresholds to luminance level with typical methodology of tachistoscopic studies of word recognition.

An experiment that measures threshold exposure duration for correct recognition at each of various set luminance levels does not fit exactly any of Kahneman's (1966a) intensity-time experiment models. The threshold duration measure, in a sense, is a latency for events required to meet a task performance criterion; there is no indication of effects of durations in excess of that required for criterion, and if time less than that required has effects, these go undetected. Reciprocity (perfect summation) holds if, in a $\log \mathrm{t}$ vs $\log \mathrm{I}$ plot, the curve has the form $\log \mathrm{t}+\log \mathrm{I}^{\mathrm{k}}=\log \mathrm{C}$, with $\mathrm{k}=1$ and $\mathrm{C}$ is a constant. However, $\mathrm{k}$ may be expected to go to infinity for some low $I$, where even continuous viewing fails to produce recognition, and $\mathrm{k}=0$, likely, beyond some high $I$ when a minimum $t$ is reached. A curve of the same general form but with $\mathrm{k}$ between 1 and 0 shows time to have the greater weight in determining criterion performance, while the opposite weighting is indicated if $k$ is more negative than 1 . In any case, should two such functions be required to fit data for a range of Is, the point of abrupt change in $k$ (even from -1 to 0$)$ must not be mistaken for the $t_{c}$ determined with other procedures and sorts of plots.

Other procedural factors introduce important variables other than intensity and time into typical tachistoscopic methods. (a) The use of the ascending series only in the method of limits produces asymmetrical biases and constitutes stimulus repetition. Graham and Ratoosh (1962) brand such measures artifactual on grounds of influences of probability of seeing factors, and Haber and Hershenson (1965) and Haber and Hillman (1966) present data showing cumulative effects of repetition to enhance clarity 
of percepts. (b) Imperfect recognition of parts of a pattern may provide for correct guesses in tachistoscopic studies, and reports based on these are indistinguishable from genuine perceptual recognition. It would be of considerable interest to have knowledge of durations at which each successive item in an array becomes recognizable. Does recognition of a nonsense word progress through stages of one letter at a time? If so, do increments in duration requirements per unit added bear a regular relation to each other and are these dependent upon luminance? Progressive determinations of threshold durations for one, two, three, etc., elements represent outcomes of adopting progressively more stringent visual performance criteria. Note that the lower the criterion the fewer the repetitions involved; thus, varying criteria may reveal differential effects of repetition. (c) Viewing conditions with instruments commonly employed provide light adaptation up to the moment of substituting the target stimulus, and then postexposure luminance is once again that of the preexposure adapting field. Substantial masking effects from such pre- and postexposure adapting conditions have been demonstrated by Kahneman (1966b). Neisser's (1967) interpretation is that the useful duration of iconic memory is reduced when a bright postexposure field superimposes upon the icon and reduces contrast in the icon.

The primary purpose of this study was to provide heretofore unavailable data on functions relating threshold duration for recognition of words to luminance levels. Such data bear upon rather than resolve the complex theoretical issues underlying the recognition process. By design, the data were to be representative of a large bulk of those already in the literature, and, therefore, typical, though perhaps questionable, psychophysical procedures and viewing conditions were used. However, the scope of the study was considerably more extensive since it included additional information on durations required to recognize increasing numbers of elements in an array, on two sizes of letters, and on arrays of Landolt "Cs." The functions established supply an answer to the theoretically relevant question of whether or not reciprocity holds between intensity and threshold exposure durations for recognition.

\section{Subjects}

\section{METHOD}

A total of 23 unpracticed undergraduate and graduate students in psychology acted as Ss in five experiments. All had normal vision or vision corrected to normal. Each $S$ provided data over several sessions spaced approximately 1 day apart.

\section{Apparatus}

Four experiments employed a standard, single-timer Gerbrands Harvard Tachistoscope that provided an adapting field of $4.4 \mathrm{~mL}$ and a test field of $5.6 \mathrm{~mL}$, as measured at the eyepiece by a Macbeth Illuminometer. The fifth experiment used the newer Model T-2B dual timer type with a measured adapting field of $5.2 \mathrm{~mL}$ and a test field of $6.6 \mathrm{~mL}$. Each tachistoscope was fitted with slotted holders between the light shield and case to allow Kodak Wratten neutral filters of equal densities to be placed in the fields of both eyes for binocular vision with natural pupils. Time measures were to the nearest $.01 \mathrm{sec}$ over a range from $.01-1.00 \mathrm{sec}$, and the adapting field was presented except during substitution of the brief test field flashes.

Nonsense word stimuli consisted of five randomly selected lower-case typewritten letters on a white background and reproduced by a Xerox process. (Test field luminance was measured with this white background paper in place, and special care was taken that letters be dark-lined and clear.) Two sizes of stimuli were used: elite type size (large) and a reduction to about $2 / 3$ size (small). Thus, heights of large letters were $1.9 \mathrm{~mm}$ or
$1.2 \mathrm{~mm}$, while small letters were 1.4 or $.88 \mathrm{~mm}$ high. Widths of lines were .24 and $.18 \mathrm{~mm}$ for large and small letters, respectively, and these subtended angles of $1.45 \mathrm{~min}$ and $1.00 \mathrm{~min}$ at the $580-\mathrm{mm}$ viewing distance in the tachistoscopes. Total length of five-letter words depended upon widths of selected letters and was between 7.02 and $7.89 \mathrm{~mm}$ for large letters and between 5.09 and $5.79 \mathrm{~mm}$ for small.

Ten sets of 10 words were prepared so that each word differed widely from each other in letter make-up and position of letters duplicated in other words, and so that each set sampled letters over the alphabet. Thus, each letter appeared an approximately equal number of times in the entire set of words used for any given condition of the experiment.

The Landolt " $\mathrm{C}$ " stimuli were groups of three 2.6-mm diam rings, $.88 \mathrm{~mm}$ apart, each with $.61-\mathrm{mm}$ wide lines and a $.35-\mathrm{mm}$ $(2.07 \mathrm{~min}$ arc) gap placed either top, bottom, right, or left on a random basis.

Test stimuli appeared with a $9 \times 1.7 \mathrm{~cm}$ opening in the center of an otherwise flat black test field. The adapting field was a $20 \times 20 \mathrm{~cm}$ white card contàining, for fixation, an outline rectangle exactly corresponding in position to the outer edges of the test field aperture.

\section{Procedure}

Each $\mathrm{S}$ was dark-adapted for $10 \mathrm{~min}$ and, thereafter, the room was kept dark except for a small shielded red lamp to illuminate $E$ 's records and dials. The $S$ was seated in front of the eyepiece, instructed to attend to each "ready" signal, and to report each letter seen in the brief flash, together with its position. Time measurements were begun at the lowest luminances pilot work showed permissible and .2 or .3 log unit increases in luminance were made after each complete recognition of the given test word, up to maximum luminance. One minute of light adaptation was given to each higher luminance.

Each S was presented with a different word (or array of "Cs") at each luminance (I) level, and different word sets were used for the two sizes within a session and for different sessions. Also, different Ss had different sets for any given condition. With each I, initially exposure time (t) was below recognition requirements, and then $.01-\mathrm{sec}$ increments in $t$ were made until one letter was correctly recognized and located, then two correct, then three, four, and five, with records being made of $t$ at which each criterion was reached. No indication of correctness was given until all five letters were recognized when filters were adjusted and the next word was brought into position.

The five experiments represented replications with variation in model of tachistoscope, partial exact replication, and variation in test object as follows:

(1) For six Ss, $t$ was determined for $1,2,3,4,5$ correct letters for each $I$ with large and with small letters in each of five sessions constituting repeated measures of $t$ but with different words. The older model tachistoscope was used.

(2) For another six Ss, a partial replication of (1) yielded t data for large letters at the five-correct criterion.

(3) Another three $\mathrm{Ss}$ in a partial replication of (1) provided $t$ values for large letters at criteria of $1,2,3,4,5$ correct.

(4) Three Ss of the group in (1) were presented a different combination of three Landolt "Cs" at each I level and $t$ was determined for 1, 2, and 3 correct locations of the gaps in each of five sessions. The older tachistoscope was used.

(5) A different group of eight Ss completely replicated (1), but using the Model T-2B tachistoscope and, thus, slightly different I levels.

\section{RESULTS}

For each $S$, the five repeated $t$ measures at each $I$ for each 


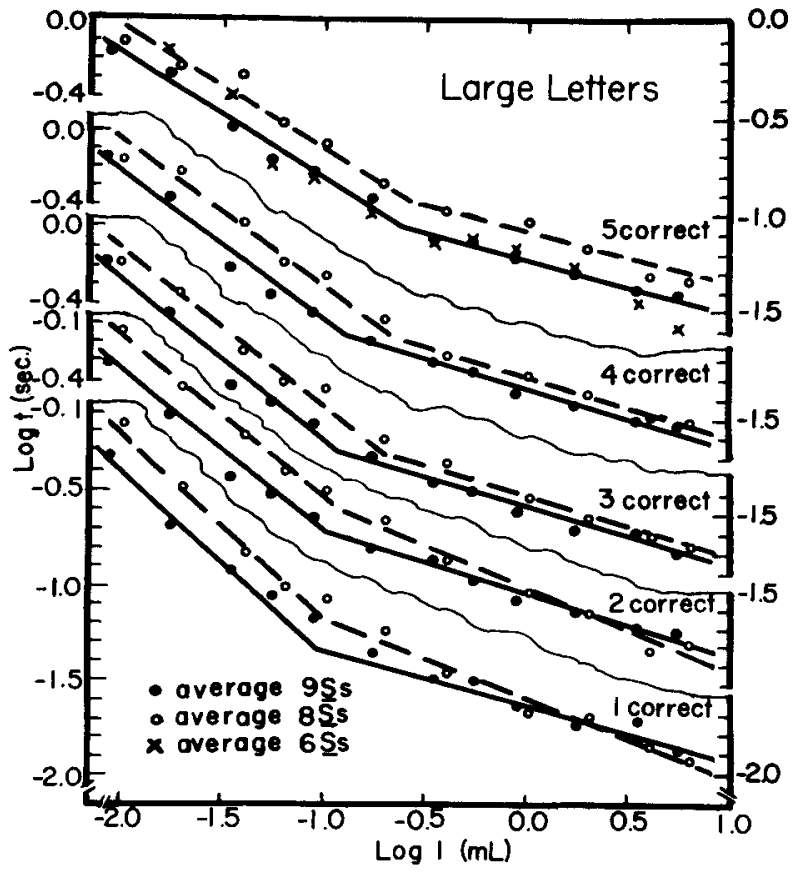

Fig. 1. $\overline{\log t}$ required for recognition of large letters at given $\log I$ (luminance) levels for each of five criteria. Note discontinuous ordinates for various curves.

criterion were averaged and converted to $\log \bar{t}$. Occasionally, the five-correct criterion was not met by $1.00 \mathrm{sec}$ at low Is, or the one- or two-correct criterion had been exceeded at $.01 \mathrm{sec}$ at high Is. In these cases of instrument limitations, $\log \bar{t}$ was based on fewer than five measures and was less reliable.

For analysis of the various conditions, data from the five experiments were considered together and data for comparable Ss under comparable conditions were pooled. While individual distributions of $t$ were fairly normal and showed moderate variability, individual differences in $\bar{t}$ were quite large and tended toward skewed distributions. Conversion to $\log \overline{\mathrm{t}}$ produced more normal distributions which were best represented by $\overline{\log \overline{\mathrm{t}}}$ and, therefore, group means were in this form. In cases of unreliable measures for an $\mathrm{S}$, these measures were not included and group means were bases on fewer than the specified $\mathrm{N}$. Thus, at extreme I levels the entire sample is not always represented.

\section{Large Letters}

Data were available for a group of nine Ss from Experiments 1 and 3 combined, for eight Ss from (5) and six Ss for five correct only from (2). Figures 1, 2, and 3 present pertinent data graphically. ${ }^{2}$ Figure 1 shows $\overline{\log } \overline{\bar{t}}$ at each $\log I$ for each criterion. Separate axes are used for different criteria to avoid overlapping of data, but note should be taken of the space-saving device involving partial superimposition of plots. The straight lines drawn were fitted by eye, dashed for eight Ss, and solid for nine Ss and for six Ss at five correct, with somewhat less weight given to less reliable data at extreme I levels. Curves for the different groups are highly similar, with the eight Ss generally showing somewhat higher $\mathbf{t s}$. In all cases, very satisfactory fits of the data are made by two curves each with the equation, $\log \mathrm{t}+\log \mathrm{I}^{\mathrm{k}}=\log \mathrm{C}$, but with abrupt changes in the constants, $\mathrm{k}$ and $C$, at certain $\log t$ and $\log I$ values. Furthermore, two sorts of changes in these constants accompany changes in criterion: (a) For lower I curves, $\mathrm{k}$ shifts from about -.66 to close to -1 from high to low criterion, while for higher I curves, $k$ changes to a lesser degree from -.25 to about -.40 . (b) Changes in $\mathrm{C}$ along with $k$ place inflection points at lower $\log t$ and $\log I$ values the lower the criterion. The change in $t$ at inflection is from about .10 to $.05 \mathrm{sec}$ and the I shifts from about .25 to $.10 \mathrm{~mL}$. The described shifts are more easily seen in Fig. 2, a composite of all curves on a single set of axes.

Separate individual data are shown in Fig. 3 since drawing conclusions about processes of individuals from group data involves dangers, and ranges of individual variation are of interest. Data for the two Ss with the highest $\bar{t}$ and the two Ss with the lowest $\overline{t s}_{\text {in }}$ the nine-Ss group are shown for the highest and lowest criteria. Greater irregularities in functions are to be expected with less reliable individual measures, but, nevertheless, double-straight-line curves fit for each $\mathbf{S}$ under each condition. Moreover, individual Ss show the same type and magnitude of shifts in $\mathbf{k}$ between higher and lower $\mathbf{I}$ curves, between high-to-low criterion, as do group data. Also, $t$ and $I$ at inflection shows comparable changes with criteria. Almost all variations for these extreme Ss are within an envelope $.5 \mathrm{log}$ unit wide and symmetrical around the group mean. Obviously, pooled data do not misrepresent individuals.

\section{Small Letters}

Data obtained from six Ss from Experiment 1 and eight Ss from Experiment 5 are presented in a manner exactly parallel to that used for large letters. Figure 4 shows that, with small letters also, the eight-Ss group required higher $\bar{t}_{s}$ than the six Ss of Experiment 1, and that the same sorts of functions that described large-letter data fit all small-letter data with two notable exceptions: the functions for eight $S$ s at the highest two criteria. For five correct, a single curve, $\log t+\log I .44=\log C^{\prime}$, fits the entire I range for eight $\mathrm{Ss}$, and, for four correct, two curves are needed, but $\mathrm{k}$ is barely detectably different for them ( .57 and -.48 ). Figure 5 is a composite of curves allowing easier comparisons between groups over criteria. As criterion is reduced, $\mathrm{k}$ shifts from about .60 to almost -1 for lower I curves, but $\mathrm{k}$ remains around -.45 to .50 for higher I curves. Changes in $\mathrm{C}$ and $\mathrm{k}$ accompanying criterion reduction do not alter the $.10-\mathrm{mL}$ I value at inflection, but $t$ decreases regularly from $.35 \mathrm{sec}$ (for six Ss) to $.10 \mathrm{sec}$. The individual data of Fig. 6 , taken from the eight-Ss group to confirm the generality of the single-line fit for the five-correct criterion, show irregularities only marginally well

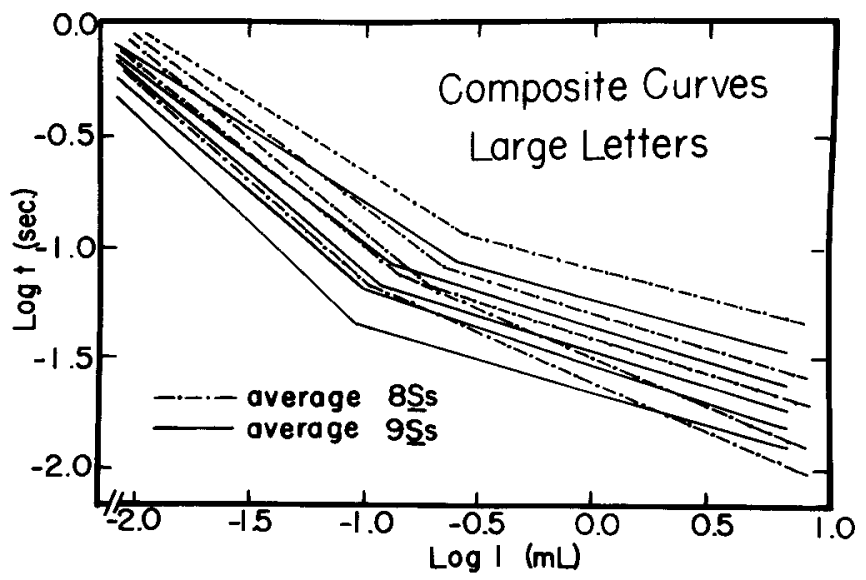

Fig. 2. The fitted average curves for the five criteria for large letters shown on a single continuous $\log t$ axis. Top dash-dot curve for eight Ss, five correct, next lower for four correct, next for three, then two, then one correct. Solid curves for nine Ss are in like order of criteria. 


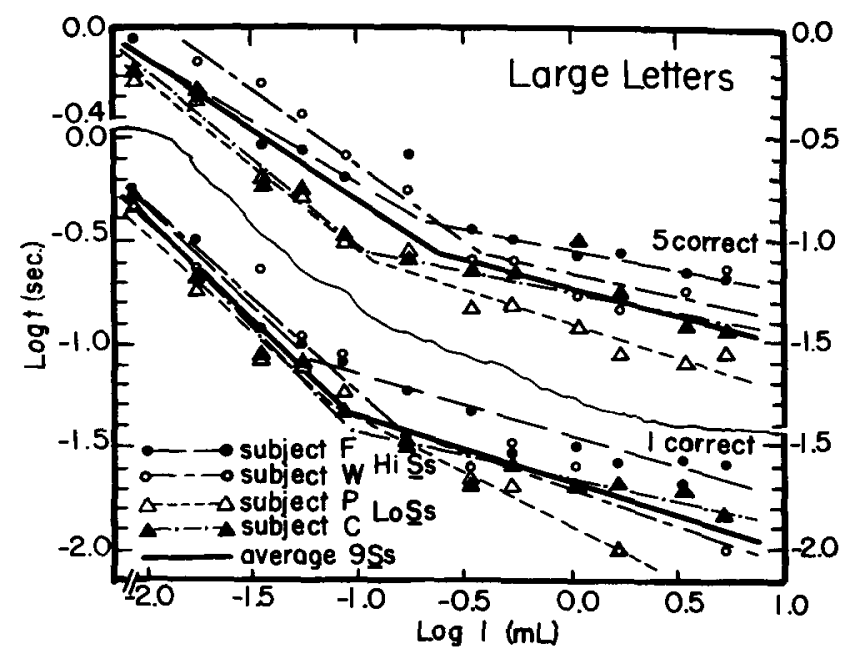

Fig. 3. Data for four individual Ss, two representing each extreme of the range of variability among Ss of nine Ss, and the fitted curves for large letters for the highest and lowest criteria. Note discontinuous ordinates.

fitted in cases. However, no $S$ gives evidence of any but a single curve with $\mathrm{k}$ between -.55 and -.41 at the high criterion. At low criterion, all Ss show double-line functions, with $\mathrm{k}=-1$ for lower Is and $\mathrm{k}$ between -.60 and -.37 for higher Is. Virtually all individual data fall within an envelope $.6 \mathrm{log}$ units wide.

Small- vs large-letter comparisons for the eight $\mathrm{Ss}$ are made clear in Fig. 7. Reduction in size (by $.55^{\prime}$ of line width) does not alter the form of equations describing $\log \mathrm{t}$ vs $\log I$ relationships, but it does alter the constants of the equations quite considerably. Changes of note are: (a) Reduction in size makes $\mathrm{k}$ less negative for lower I curves at high criteria, but $k$ is not

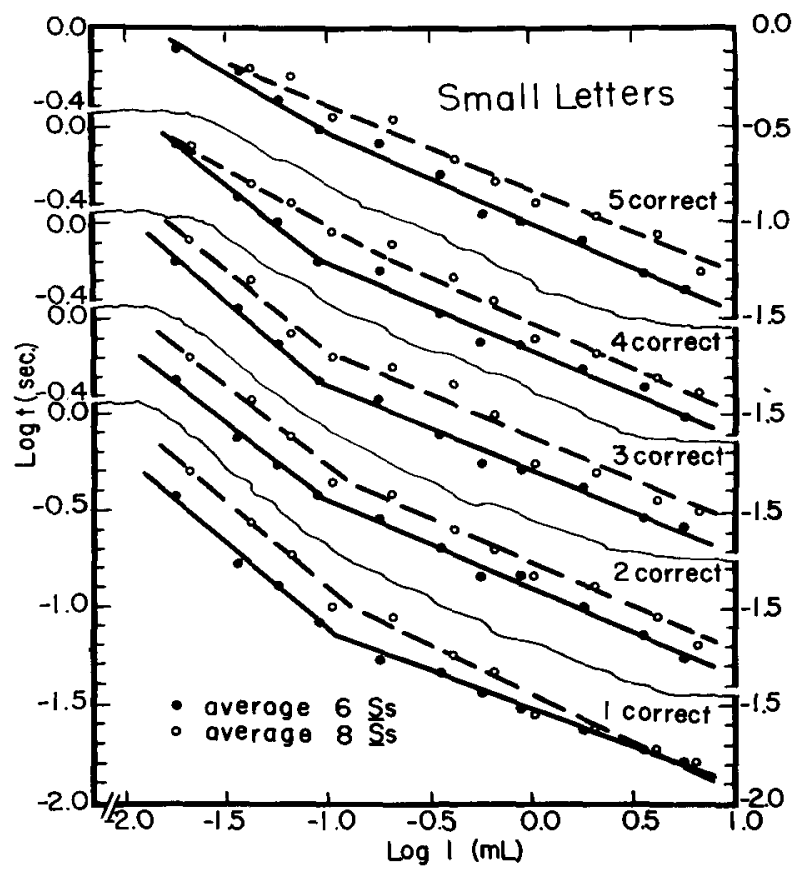

Fig. 4. $\overline{\log \bar{t}}$ required for recognition of small letters at given $\log$ I (luminance) levels for each of five criteria. Note discontinuity of ordinates of curves.

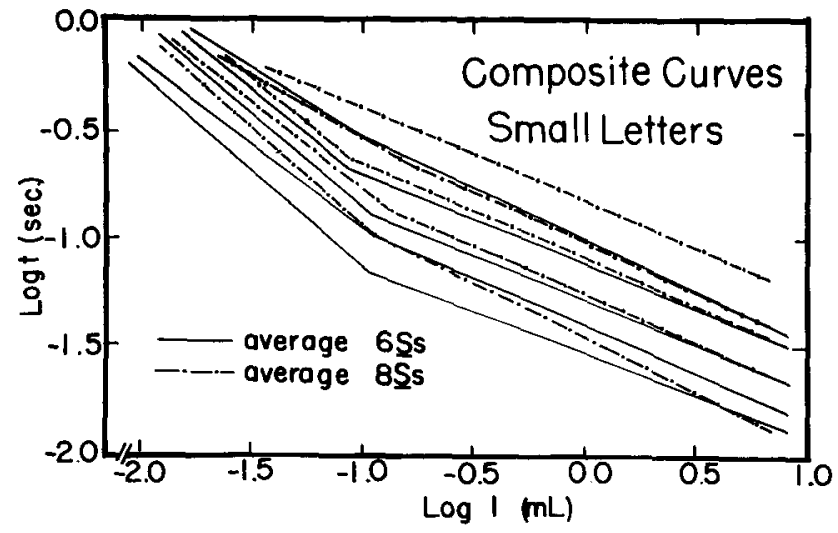

Fig. 5. The fitted average curves for the five criteria for small letters shown on a single continuous $\log t$ axis. Dash-dot curves for eight $\mathrm{Ss}$ in order of descending criteria from topmost to bottom, as are solid curves for six Ss.

affected (around -1) for low criterion. (b) Size reduction makes $\mathrm{k}$ more negative for higher I curves (still far from -1 , though), and criterion variation no longer effects regular shifts in $\mathbf{k}$. (c) With size reduced, $\mathrm{k}$ and $\mathrm{C}$ changes no longer produce lowering of I at inflection over decreasing criteria, although there is somewhat greater reduction in $t$ values for inflection points.

\section{Letters vs " $C$ "}

The five $t$ measures for each $I$ at each criterion for gap location were averaged for each of the three Ss of Experiment 4. Group means were computed as above $(\overline{\log \bar{t}})$. Comparable treatment was given large-letter data for 1, 2, and 3 correct recognitions obtained for these same Ss in Experiment 1. Figure 8 shows the highly similar results for "Cs" and letters. These particular Ss deviate some from the nine-Ss group as a whole in showing $\mathrm{k}$ for lower Is to be -1 , or more negative, for all three criteria with letters. However, within-Ss comparisons between tasks make the major point of generality of $\log t$ vs $\log I$ relationships obtained with nonsense words.

\section{DISCUSSION}

Not unexpectedly, $t$ has been seen to depend upon I, stimulus size, and stringency of criterion. But there are interactions among these variables that determine $t$. Basically, the $t$ vs I relationship showed proportionate reductions of $\log t$ with increases in $\log I$ describable by the equation, $\log t+\log I^{k}=\log C$. However, different constants of proportionality $(\mathrm{k})$ held for lower Is than for higher Is, the exact value of $\mathrm{k}$ (and also $\mathrm{C}$ ) depending upon size of stimuli and stringency of criterion.

Reciprocity between $t$ and $I$ is the exception rather than the rule; it holds only with very low criteria (one or two correct elements out of five) and low Is (from $.01-10 \mathrm{~mL}$ ). At higher criteria for this range of Is, log $t$ reduction does not keep pace with $\log I$ increases, and, thus, time appears to have a greater weight than luminance in determining recognition. For higher Is (.10 to almost $7 \mathrm{~mL}$ ), some partial summation is clearly evidenced at all criteria $(k \neq 0)$; one $\log$ unit increase in I permits between .25 and $.50 \mathrm{log}$ unit decrease in $\mathrm{t}$. These several findings have proved replicable over different groups, over Ss within groups, and over tachistoscopes. Two sizes of letters and Landolt "Cs" show the same sorts of relationships, although size affects $\mathrm{k}$ and $\mathrm{C}$ in certain respects.

It remains to be discovered to what extent the present results are traceable to either psychophysical procedures employed, or 


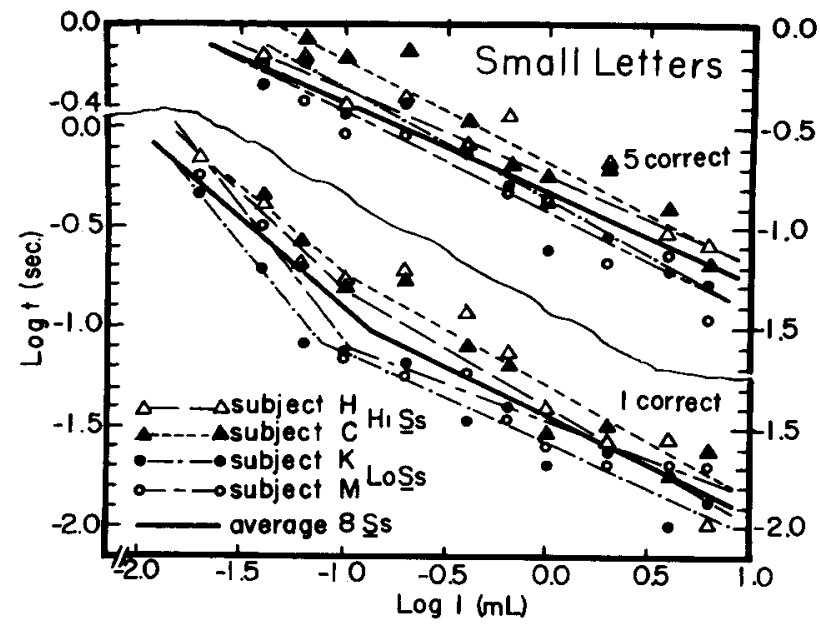

Fig. 6. Data for four individual Ss, two representing each extreme of the range of variability among eight $S s$, and the fitted curves for small letters for the highest and lowest criteria. Note discontinuous ordinates.

light adapting fields before and after target, or both. If there is general reciprocity which is obscured by such procedural factors, they are indeed potent factors demanding considerable attention.

Criterion variations produced a strong effect and, it will be remembered, variation in numbers of repetitions of stimuli accompanies the criterion stringency variable. If a low criterion has the repetition factor all but eliminated, and higher criteria have substantial influences from repetition, perhaps reciprocity at the low criterion shows the unconfounded $t$ vs I relationship. If so, why does reciprocity not hold for higher Is? Pre- and postexposure adapting field conditions may supply an answer. Adapting-field luminance varies with test-field luminance with filters placed at the eyepiece. Suppose masking effects from adapting fields are negligible below a certain brightness, and are determining factors above that $I$. Then the higher I data would be contaminated by masking effects, plus repetition effects at higher criteria, while lower I data would be subject to cumulative repetition effects only, above the lowest criterion. These speculations can be no more than tentative, though they have a certain plausibility. The effects of size of stimuli and of criterion

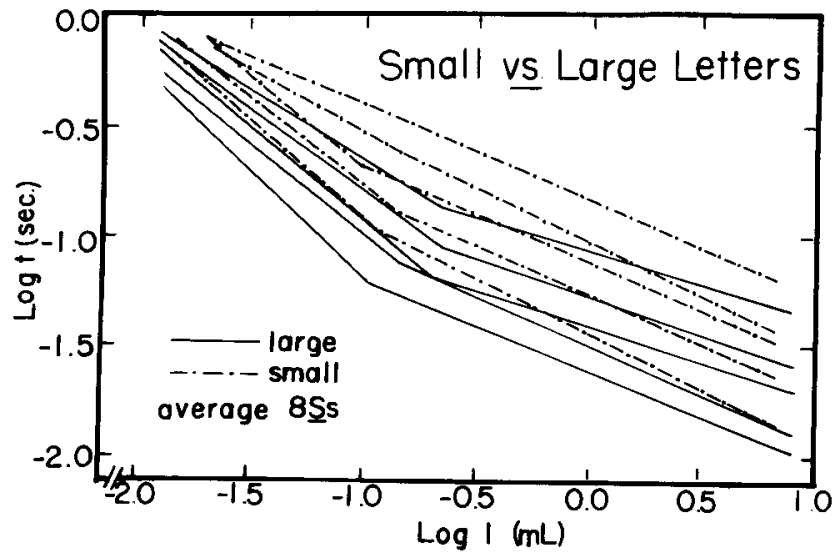

Fig. 7. Comparison of fitted curves for small and large letters for eight Ss. Dash-dot small-letter curves in order of descending criteria from topmost to bottom, as are solid line large-letter curves. upon the value of $I$ at which inflection points come present some complications for these proposed relationships among the various influences.

The alternative to attributing results to artifacts of procedure is to hypothesize that visual recognition is a process in which time and luminance relate to each other in a way different from simpler brightness discrimination and acuity processes. There is some precedent for and reasonableness to such a position. Liebowitz and Bourne (1956) note an influence of time on perceived shape that is in addition to the role time plays in the reciprocity relationship. Maximum tendency toward shape constancy required more than the usual critical duration and, while duration and luminance were parameters, the influence of the parameters was not a simple reciprocity. Kaswan and Young (1963), employing complex displays for visual differentiation, present data leading them to postulate a two-phase temporal process, one for mere detection of stimuli and following the reciprocity law, and another additional one for cases where spatial relations among elements of an array are relevant to the visual task, this latter phase requiring more exposure time the higher the spatial discrimination demands. Kahneman (1966b)

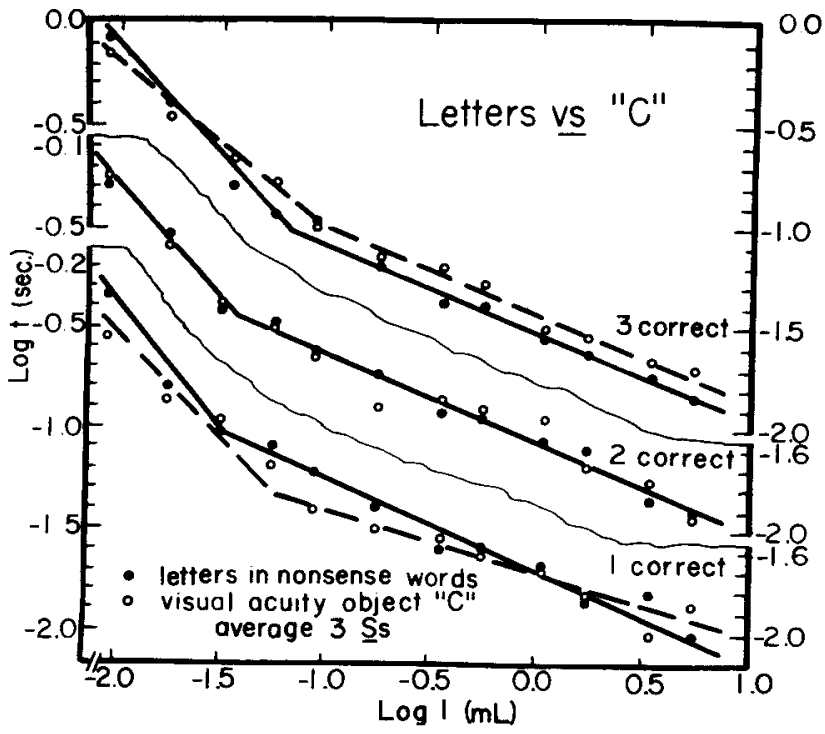

Fig. 8. Comparison of $\overline{\log \overline{\mathrm{t}}}$ for large-letter word recognition and for Landolt "Cs" at each $\log I$ for three criteria. Note ordinate discontinuity.

found with his method that reciprocity holds only when the flash that illuminates the target is added to an unchanging background, otherwise time is more important than intensity in determining performance. He reinterprets Kaswan and Young's data as due to masking effects. In addition, Kahneman and his coworkers note cases where reciprocity holds, but they conclude that long critical durations imply central and not purely sensory contributions. Kahneman (1964) reports that the Landolt " $C$ " differs markedly from brightness discrimination and grating acuity in this regard, and Kahneman and Norman (1964) found identification of triads of digits showed reciprocity up to $.35 \mathrm{sec}$ compared to $t_{c}$ of $.10 \mathrm{sec}$ for brightness discrimination for the same Ss.

None of the above studies reports findings very closely related to the present ones of double relationships both showing partial summation in all but very restricted conditions. But they concur in showing relationships between time and luminance beyond $.05-.10 \mathrm{sec}$, which appears a reasonable limit for sensory events, and they agree in affording time an extra weight in the totai 
visual process. There seems to be merit te the conclusion that tachistoscopic studies, including the present one, do not simply involve the summation of intensity over time for the purely sensory events, but instead indicate time requirements of a more central information processing. Clearly, additional investigations are needed for a full understanding of how time and luminance affect all the processes involved.

A word is in order on how acuity demands (size) influence absolute values of $t$. Increasing acuity requirements from .7 to 1.0 does not increase $t$ uniformly over Is; little effect was found at both extremes $(.01$ and $6.6 \mathrm{~mL})$, but .2 to $.4 \log$ units higher of $\mathrm{t}$ was needed for the higher acuity at .3 or $.4 \mathrm{~mL}$. Maximum increases in $\log \mathrm{t}$ were roughly the size of the alteration in $1 / \alpha$.

To answer the question of whether or not recognition of an array progresses in a stepwise fashion, generally Ss reported the addition of one letter at a time to the recognized group. This is a qualified "yes," since on occasions "leaps" of two or three letters were added over the $.01 \mathrm{sec}$ increment in $t$. Overall, curves for progressively higher criteria were readily obtained with few trials yielding no measure for a certain criterion. As for size of $\log t$ steps between criterion levels, these were not uniform over Is; they generally increased up to about $.10 \mathrm{log}$ unit intervals at the breaking points of curves, and then at higher Is the increments were variable and somewhat smaller. Intervals tended to be slightly larger between higher criterion levels at which greater repetitions of stimuli might have had beneficial influences.

Important implications for practices of treating, combining, and comparing data from typical tachistoscopic studies of visual recognition are to be found in the fact that absolute measures of threshold duration are not related linearly over luminances, over sizes of letters, or over performance criteria. These previously unknown empirical relations should have significance for such researches as well as serving to delineate questions of theoretical interest about underlying visual recognition processes.

\section{REFERENCES}

GRAHAM, C. H., \& COOK, C. Visual acuity as a function of intensity and exposure-time. American Journal of Psychology, 1937, 49, 454-461.

GRAHAM, C. H., \& RATOOSH, P. Notes on some interpretations of sensory psychology, perception, and behavior. In S. Koch (Ed.), Psychology: A study of a science. Vol. 4. New York: McGraw-Hill, 1962. Pp. 483-514.

HABER, R. N., \& HERSHENSON, M. The effects of repeated brief exposures on growth of a percept. Journal of Experimental Psychology, $1965,69,40-49$.

HABER, R. N., \& HILLMAN, E. R. The effect of repetition on perception of single letters. Perception \& Psy chophysics, 1966, 1, 347-350.

HUNTER, W. S., \& SIGLER, M. The span of visual discrimination as a function of time and intensity of stimulation. Journal of Experimental Psychology, 1940, 26, 160-179.

KAHNEMAN, D. Temporal summation in an acuity task at different energy levels: A study of determinants of summation. Vision Research, $1964,4,557-566$.

KAHNEMAN, D. Exposure duration and effective figure-ground contrast. Quarterly Journal of Experimental Psychology, 1965, 17, 308-314.

KAHNEMAN, D. The time-intensity reciprocity in acuity as a function of luminance and figure-ground contrast. Vision Research, 1966a, 6, 207-215.

KAHNEMAN, D. Time-intensity reciprocity under various conditions of adaptation and backward masking. Journal of Experimental Psychology, $1966 \mathrm{~b}, 71,543-549$.

KAHNEMAN, D., \& NORMAN, J. The time-intensity relation in visual perception as a function of observer's task. Journal of Experimental Psychology, 1964, 68, 215-220.

KASWAN, J., \& YOUNG, S. Stimulus exposure time, brightness, and spatial factors as determinants of visual perception. Journal of Experimental Psychology, 1963, 65, 113-123.

LEIBOWITZ, H., \& BOURNE, L. E., JR. Time and intensity as determinants of perceived shape. Journal of Experimental Psychology, $1956,51,277-281$.

LIT, A. Visual acuity. In Annual Review of Psychology. Vol. 19. Palo Alto, Calif., 1968.

NEISSER, U. Cognitive psychology. New York: Appleton-Century-Crofts, 1967.

RIGGS, L. A. Visual acuity, In C. H. Graham, N. R. Bartlett, J. L. Brown, Y. Hsia, C. G. Mueller, and L. A. Riggs, Vision and visual perception. New York: Wiley, 1965.

\section{NOTES}

1. Address: Psychology Department, State University College, 1300 Elmwood Ave, Buffalo, New York 14222.

2. Tables of numerical data for these and all graphs to follow are on file with Perception \& Psychophysics.

(Accepted for publication February 5, 1969.) 